\title{
The ATLAS Fast Track Simulation Project fatias
}

Šárka Todorova, Tufts U. on behalf of ATLAS Collaboration

\section{Outline:}

$>$ Introduction

$>$ Motivation

$>$ Fatras ID (Inner Detector)

$>$ Fatras MS (MuonSpectrometer)

$>$ Fatras Combined

$>$ Validation \& Performance

$>$ Summary

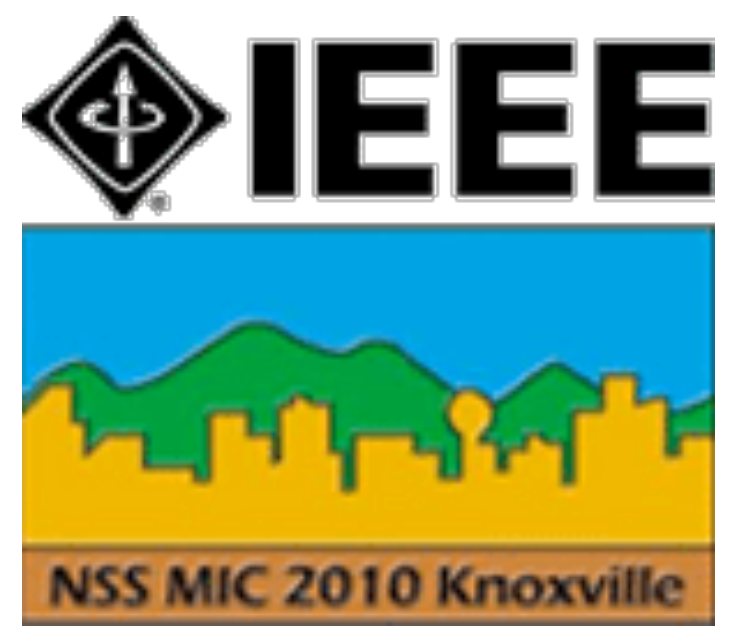




\section{ATLAS Collaboration}

\section{atias}

Šárka Todorova

IEEE2010

\section{Introduction}

Motivation

Fatras ID

Fatras MS

Fatras Combined

Validation \&

performance

Summary

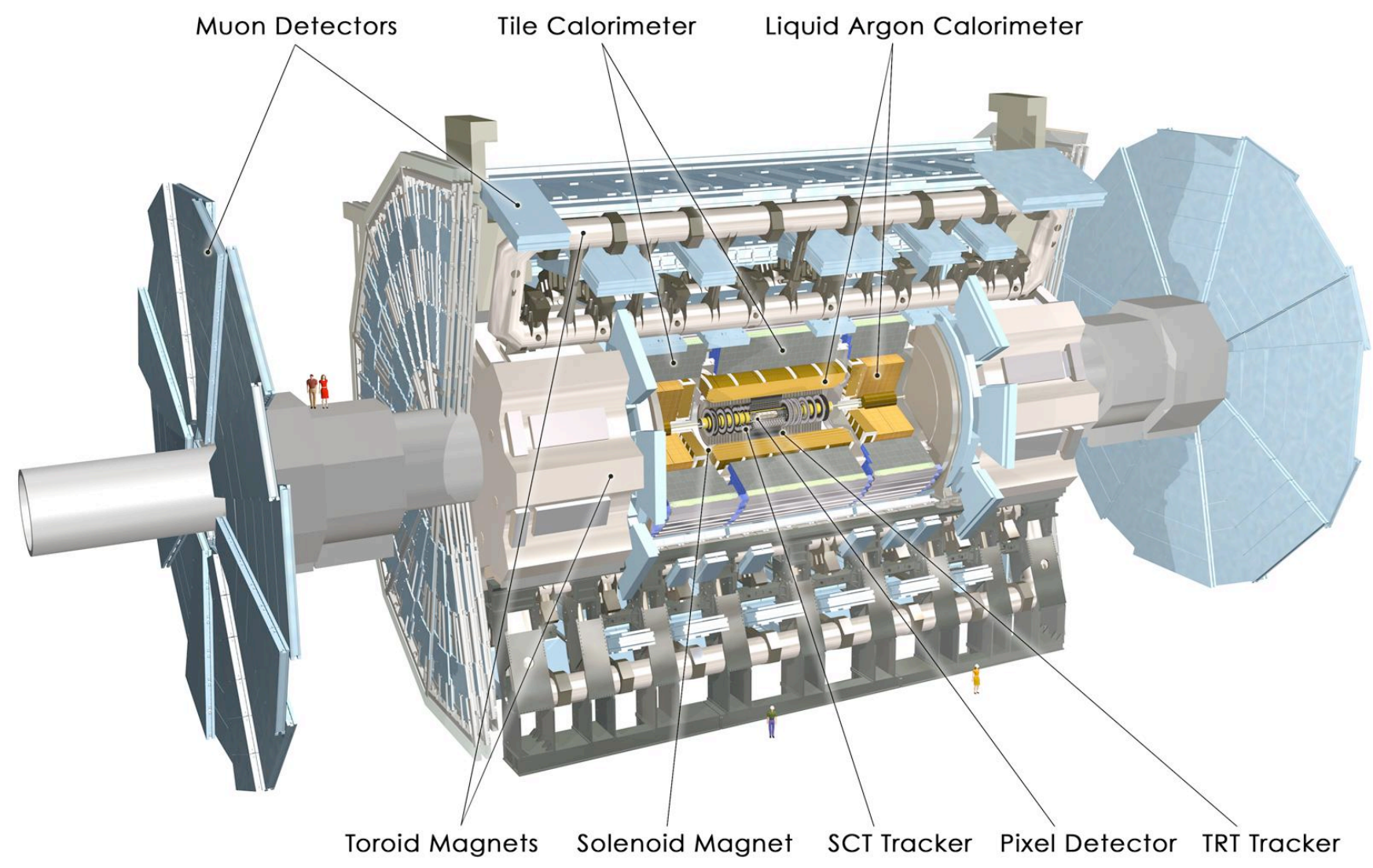


fatias

\section{Motivation}

To a large extent, FATRAS is a by-product ( and an obvious application ) of ATLAS track reconstruction tools

$\rightarrow$ material service \& extrapolation for reconstruction purpose

" what is needed to reconstruct, may be enough to simulate "

Add the necessary minimum to reproduce fully simulated and reconstructed quantities : secondary interaction smearing noise

Impose momentum cuts : typically, we use a low momentum cut $>150 \mathrm{MeV}$ (adjustable)

Large number of switches regulating the performance Possibility to tune the efficiency of detector response (hit creation) 


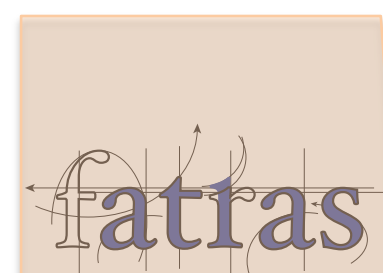

Šárka Todorova

IEEE2010

Introduction

Motivation

Fatras ID

Fatras MS

Fatras Combined

Validation \&

performance

Summary

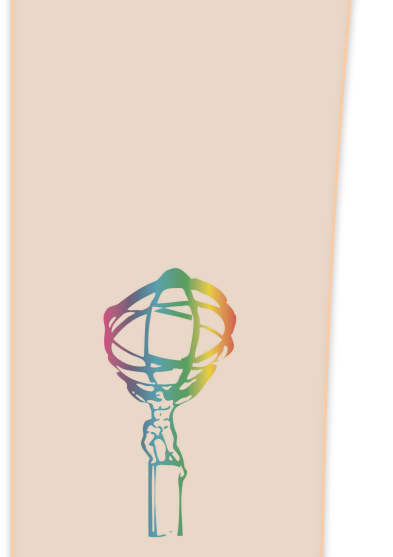

3

\section{ATLAS Inner Detector}

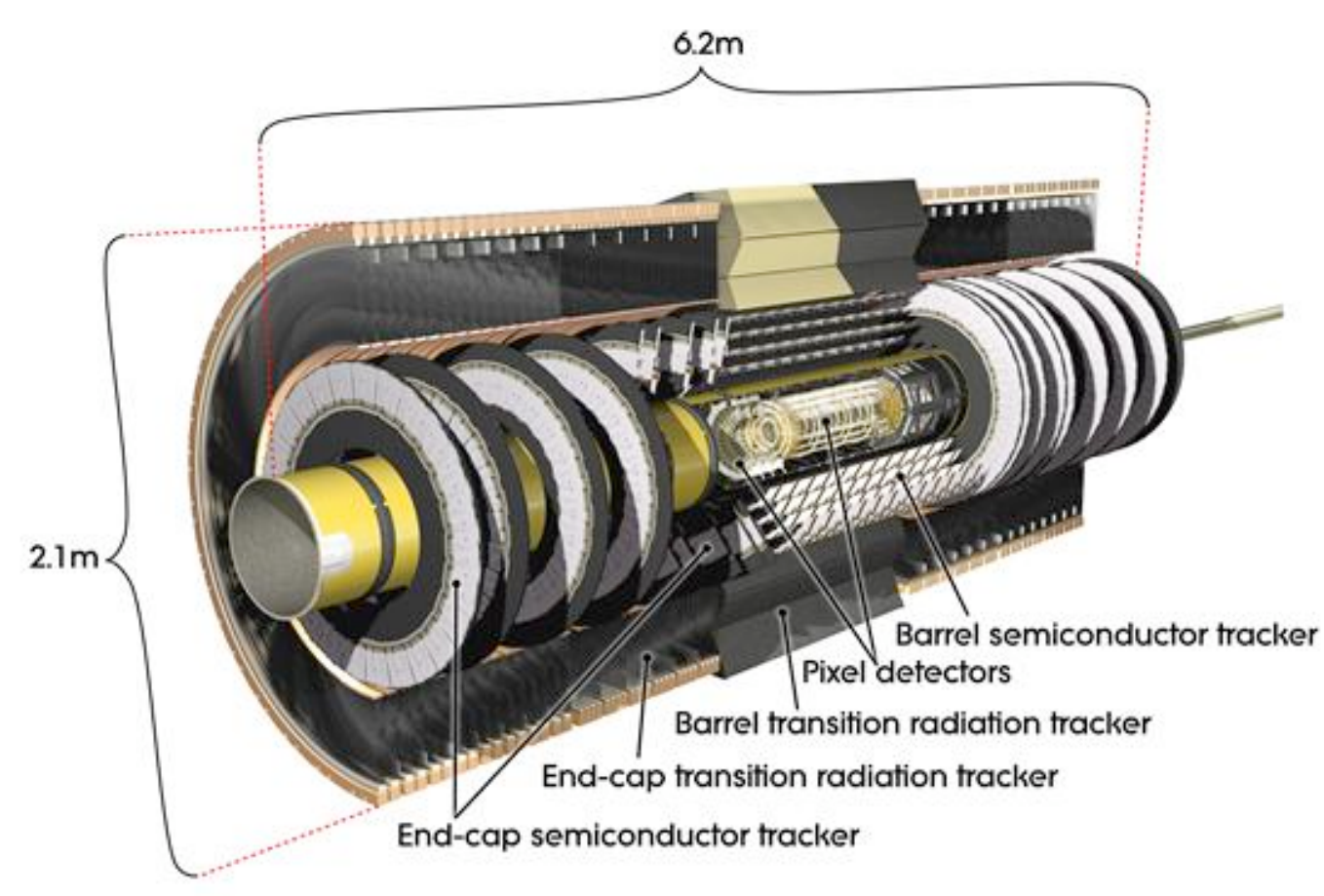

silicon Pixel

$\approx 80$ million pixels

$10 \mu \mathrm{m}(R \phi) / 115 \mu \mathrm{m}(\mathrm{Z})$

3 measurements/track $+\quad$ SCT silicon strips

$\approx 6$ million Si strips

$17 \mu \mathrm{m}(\mathrm{R} \phi) / 580 \mu \mathrm{m}(\mathrm{Z})$

4(double) meas./track
TRT drift tubes

$\approx 300000$ straw tubes $130 \mu \mathrm{m}(R \phi)$

30 meas./track 


\section{FATRAS strategy in ID}

\section{atias}

Sárka Todorova

IEEE2010

Introduction

Motivation

Fatras ID

Fatras MS

Fatras Combined

Validation \&

performance

Summary

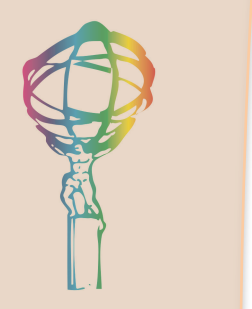

Simple (tracking) geometry:

$3+2 \times 3$ Pixel layers,

$4+2 \times 9$ SCT layers,

Track Extrapolation as

in reconstruction:

robust navigation, implicit

material effects ( energy loss )

+ multiple scattering (smearing)

+ decay of unstable particles

+ photon conversion

+ (electron) bremstrahlung

+ hadronic interactions ( simplified parametrization )

Intersections of track with sensitive surfaces used to produce hits:

+ noise added

+ clusterization

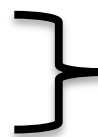

electronic signal simulation ("digitization")

Link to Conditions Database takes into account detector status (inactive channels)
Map of hadronic interactions

mulation

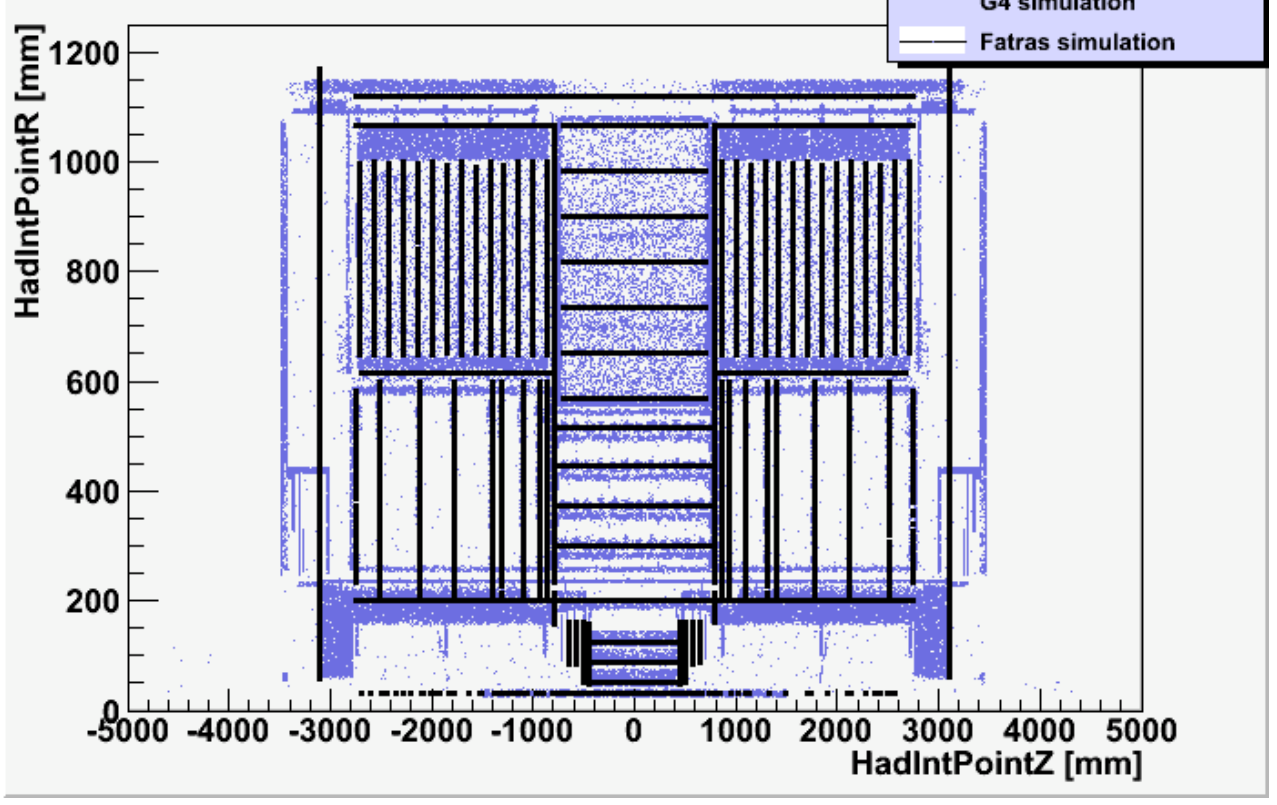

Beam spot service implemented. 


\section{fatias}

\section{Fatras ID validation: hit rate in reconstructed minimum bias sample}

\section{Pixels}

Fatras Combined

Validation \&

performance

Summary

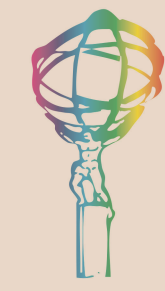

5
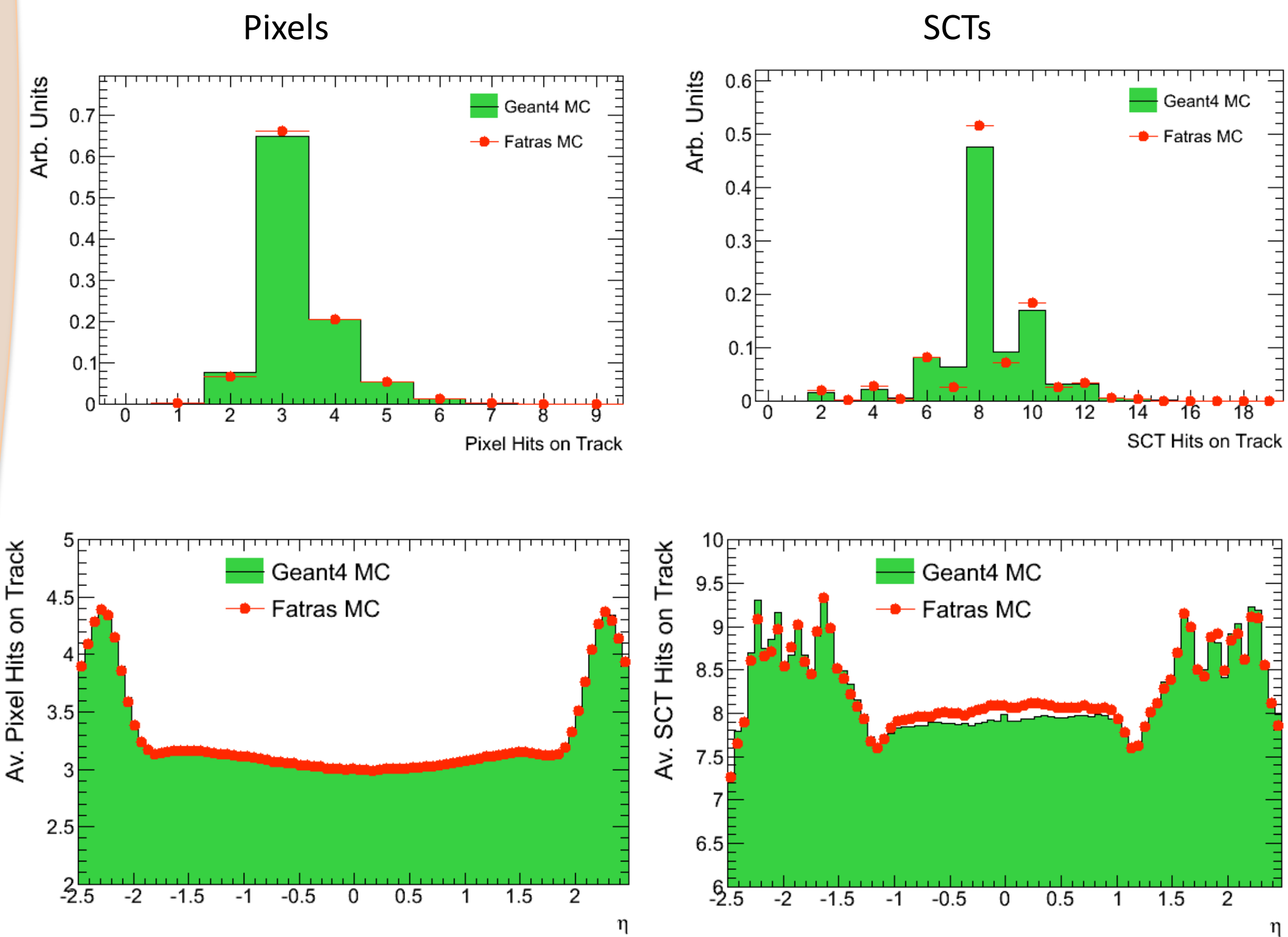


\section{fatias}

Fatras ID validation: reconstructed impact parameter

(minimum bias sample)

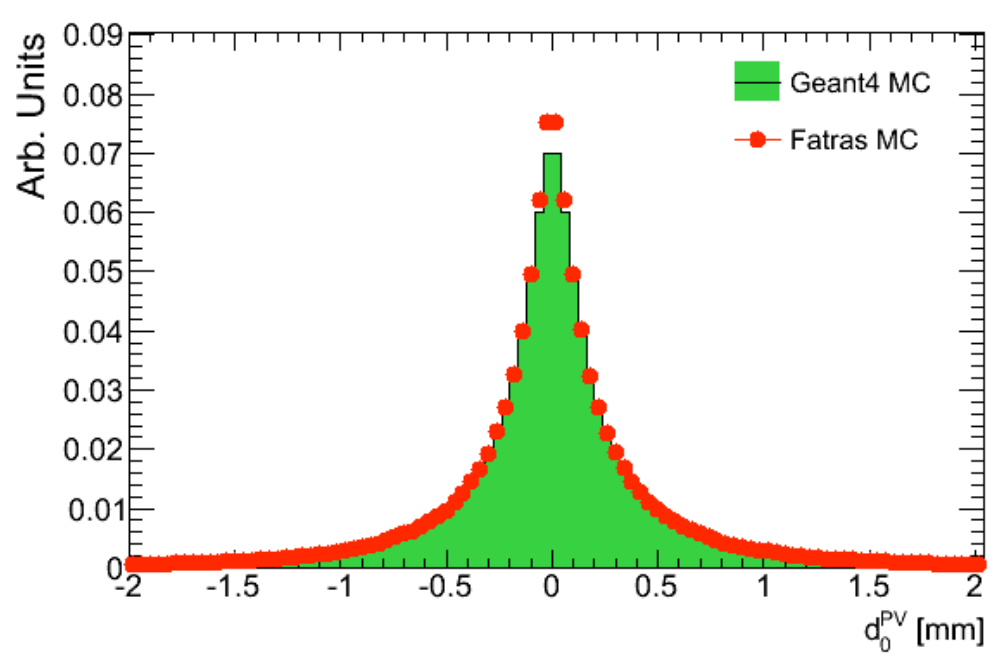

Timing performance:

( minimum bias sample )

Full simulation + digitization Fatras simulation (+digi)
A bit narrower in Fatras compared to full simulation A good agreement nevertheless with typical selection cuts ( $1-2 \mathrm{~mm}$ )

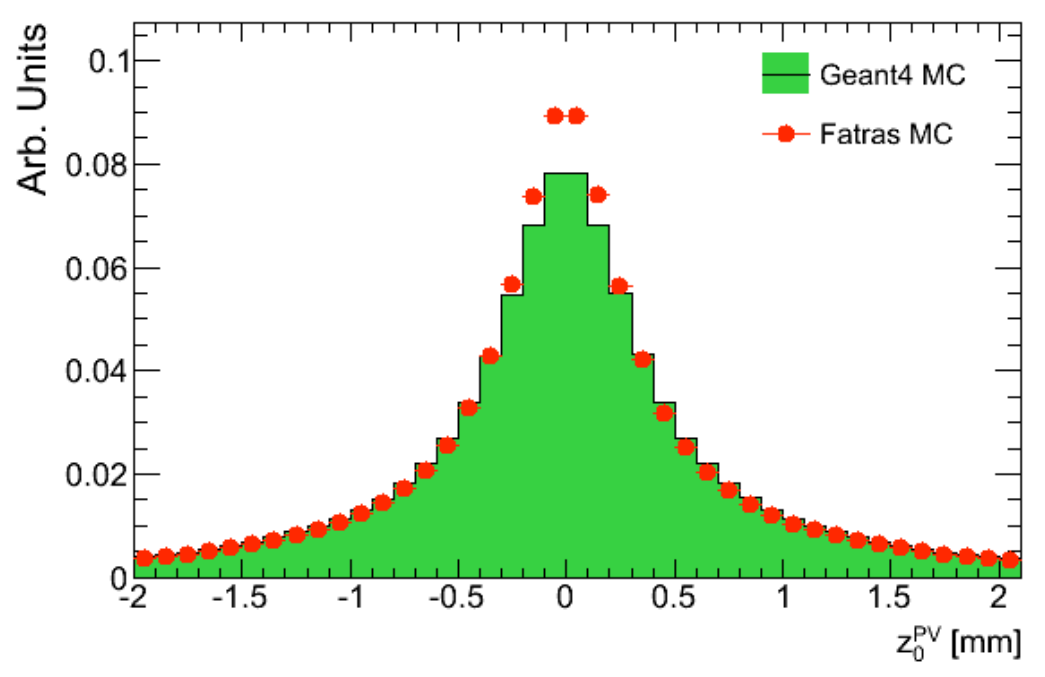

$>1$ minute / event

$<1 \mathrm{sec} /$ event 


\section{ATLAS MuonSpectrometer}

fatras

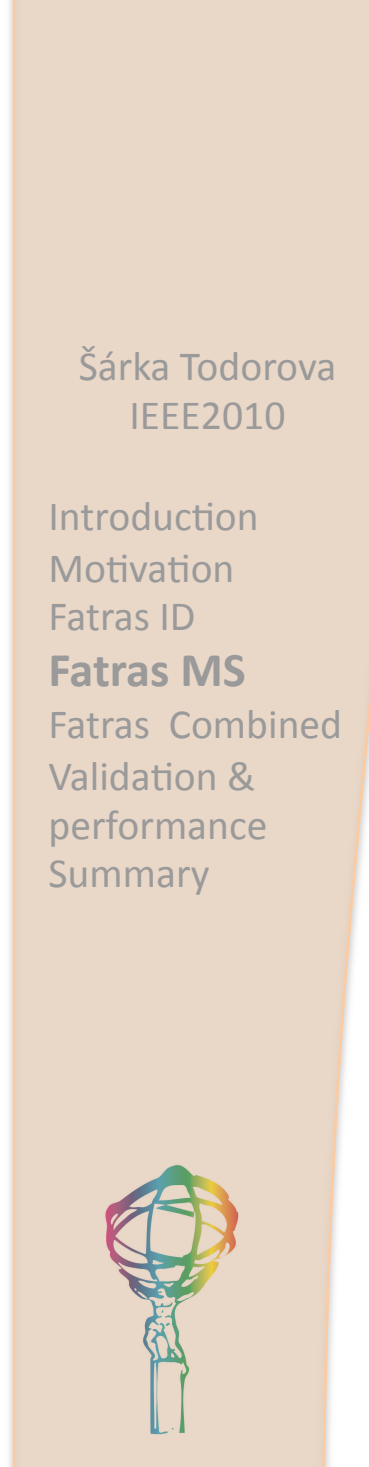

7

\section{Endcap:}

2 'big wheels' (MDT, TGC) 1 'small wheel' (MDT, CSC)

Barrel: 3 layers of stations (inner, midle, outer) (MDT, RPC)

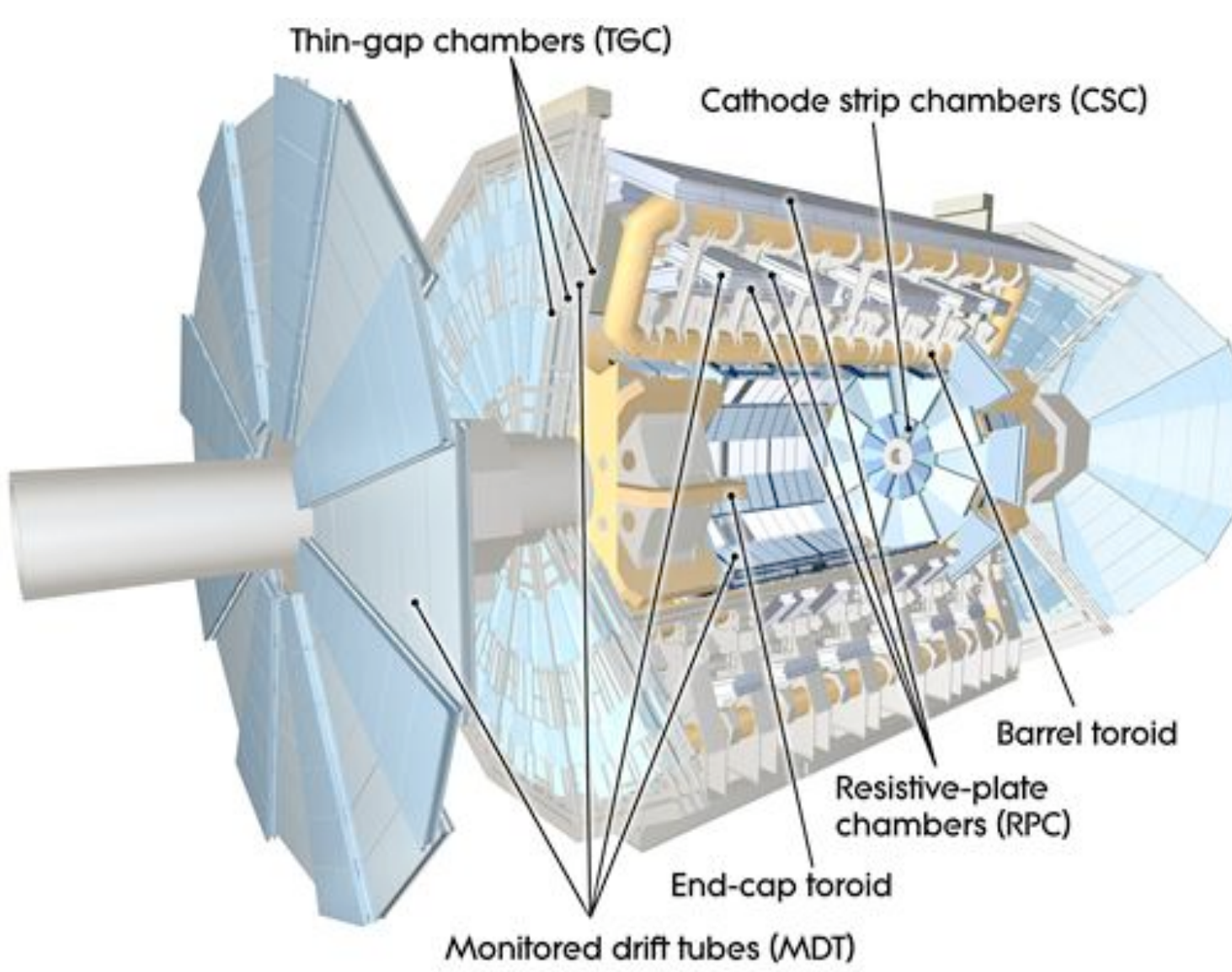

Monitored drift tubes(MDT) $<100 \mu m(\eta) \quad(\sim 1 \mathrm{~cm}$ in $\phi)$

Resistive-plate chambers(RPC) $(\sim 1 \mathrm{~cm}$ in $\eta, \phi)$
Thin-gap chambers(TGC) 


\section{fatras}

Šárka Todorova

IEEE2010

Introduction

Motivation

Fatras ID

Fatras MS

Fatras Combined

Validation \&

performance

Summary

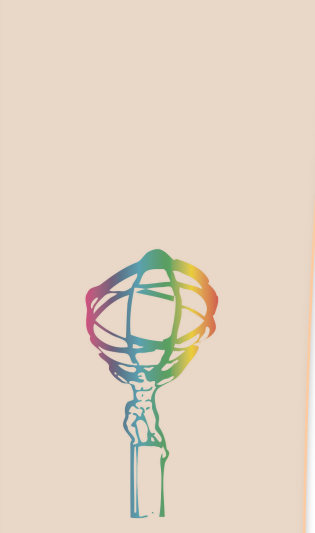

8

\section{FATRAS strategy in MS}

Simplified active geometry ( example: middle Barrel station) material scan for G4/Fatras simulation

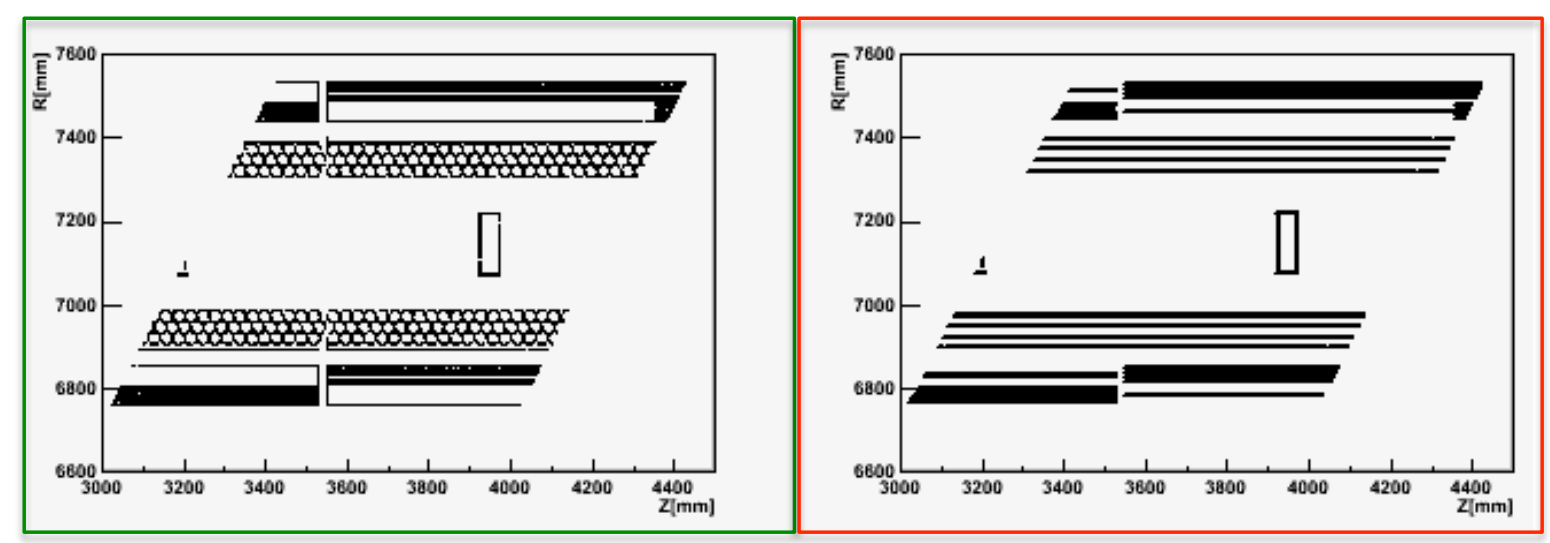

Exact transcript of inert material dense volumes->boundary surfaces

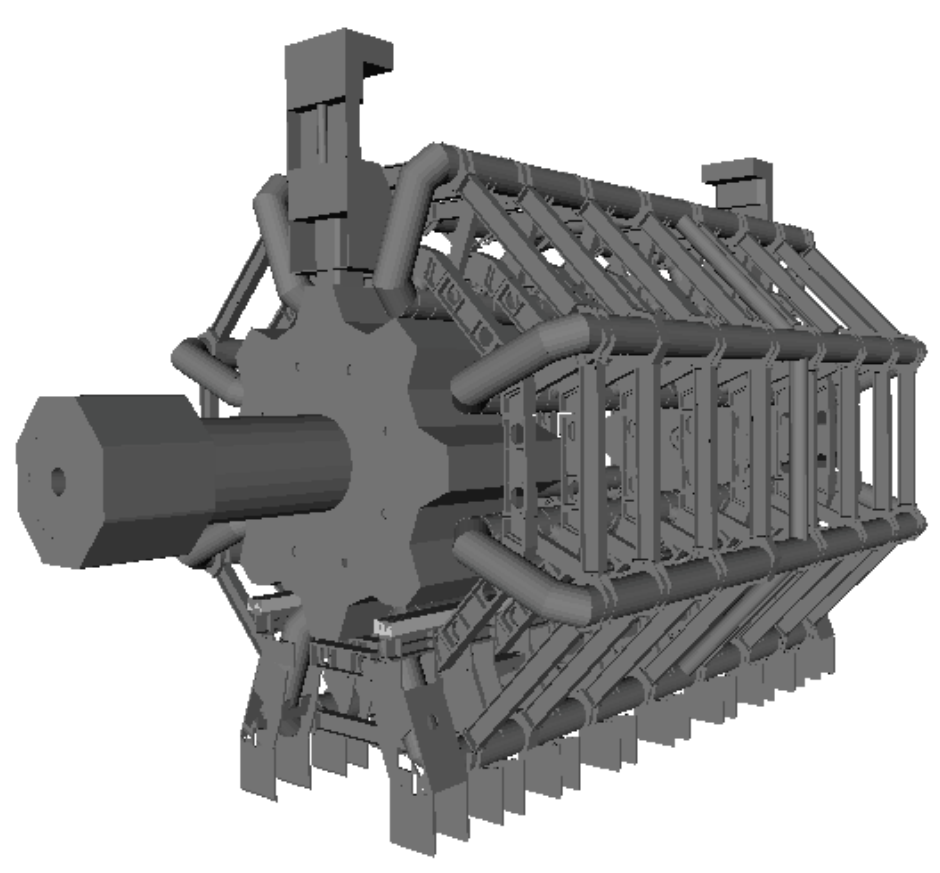

Muons: no secondary interactions ( energy loss and smearing applied)

STEP propagator :

- build-in material effects and error propagation

- adapted for efficient navigation within ATLAS Reconstruction Geometry Model

Create simulated hits collections. Use standard digitization chain. 


\section{fatias}

Šárka Todorova

IEEE2010

Introduction

Motivation

Fatras ID

Fatras MS

Fatras Combined

Validation \&

performance

Summary

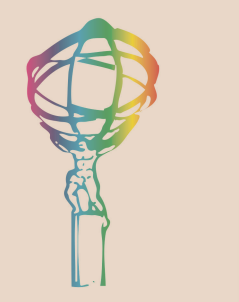

9
Fatras MS validation: hit rate in single muon sample

Muon station hit occupancy: good agreement between primary simulated hit rate ( Fatras vs. G4)

There are no secondary hits in Fatras ....
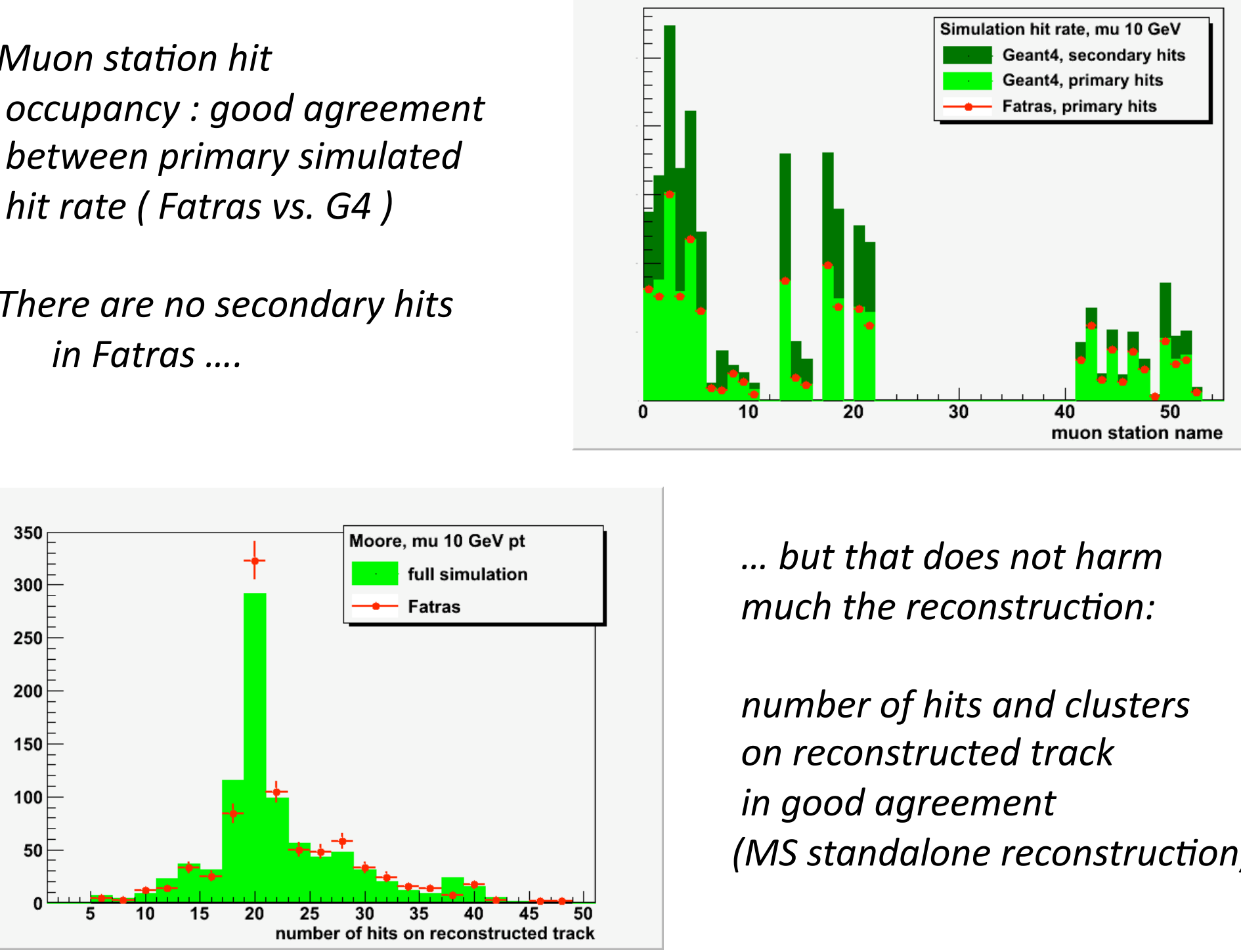

... but that does not harm much the reconstruction:

number of hits and clusters on reconstructed track in good agreement (MS standalone reconstruction) 


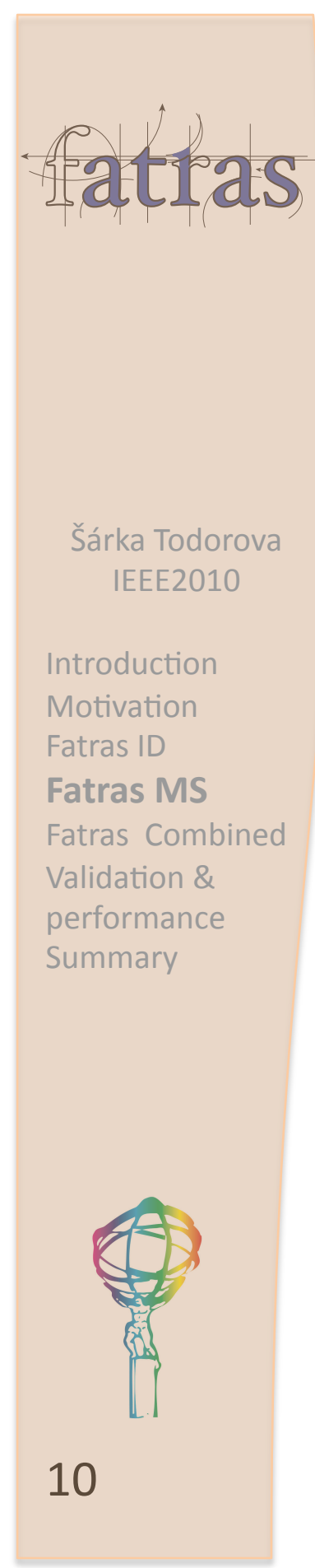

Fatras MS validation: pulls and efficiencies in single muon sample
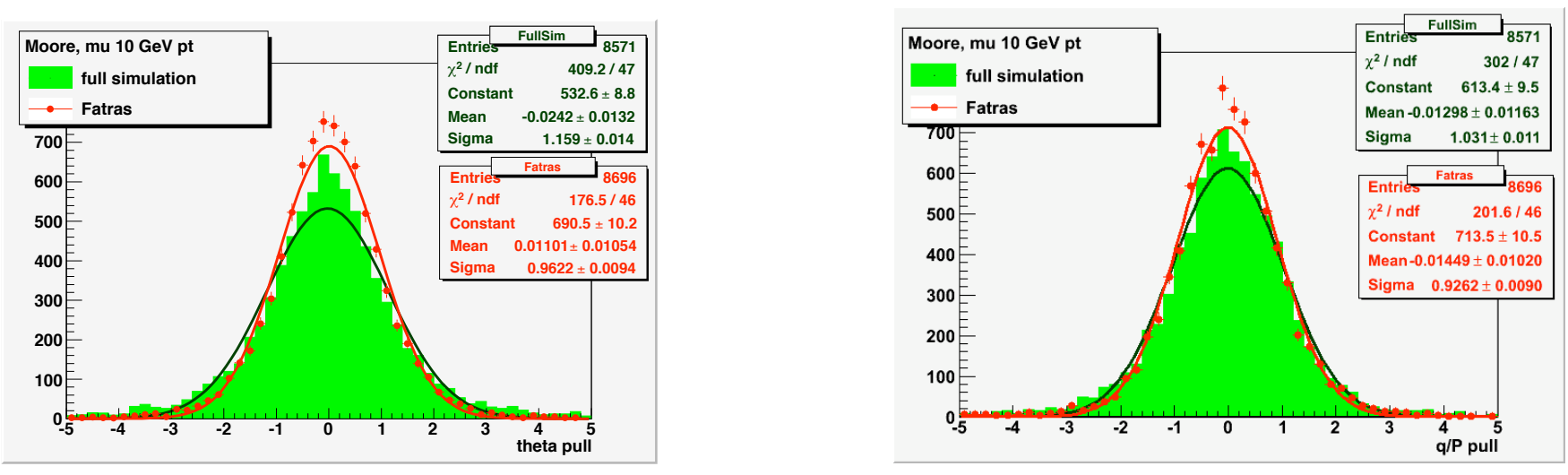

Pulls of reconstructed muon parameters (at MS entrance) slightly narrower in Fatras ( 10\%)

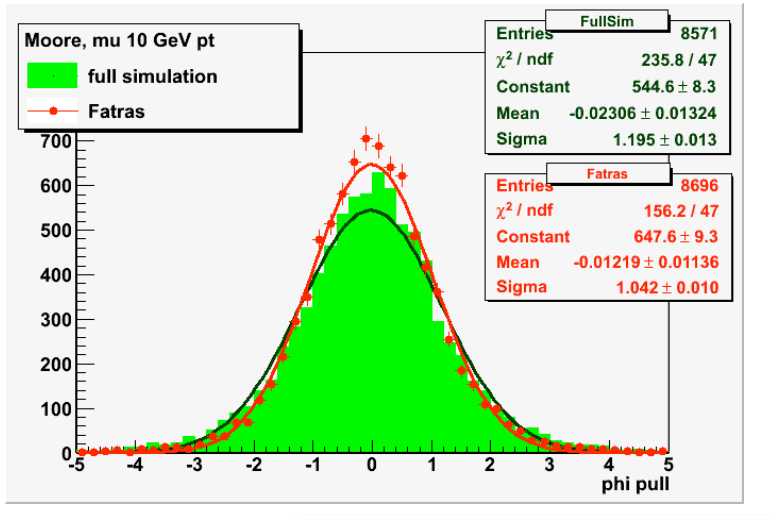

$$
\frac{\left(p_{\text {sim }}-p_{\text {rec }}\right)}{\sigma\left(p_{\text {rec }}\right)}
$$

Reconstruction efficiency in very good agreement except for very large $n$ (due to missing showers?)

\section{Timing performance:}

FatrasMS 10 times faster than $G 4$ (gen.event->sim.hits)

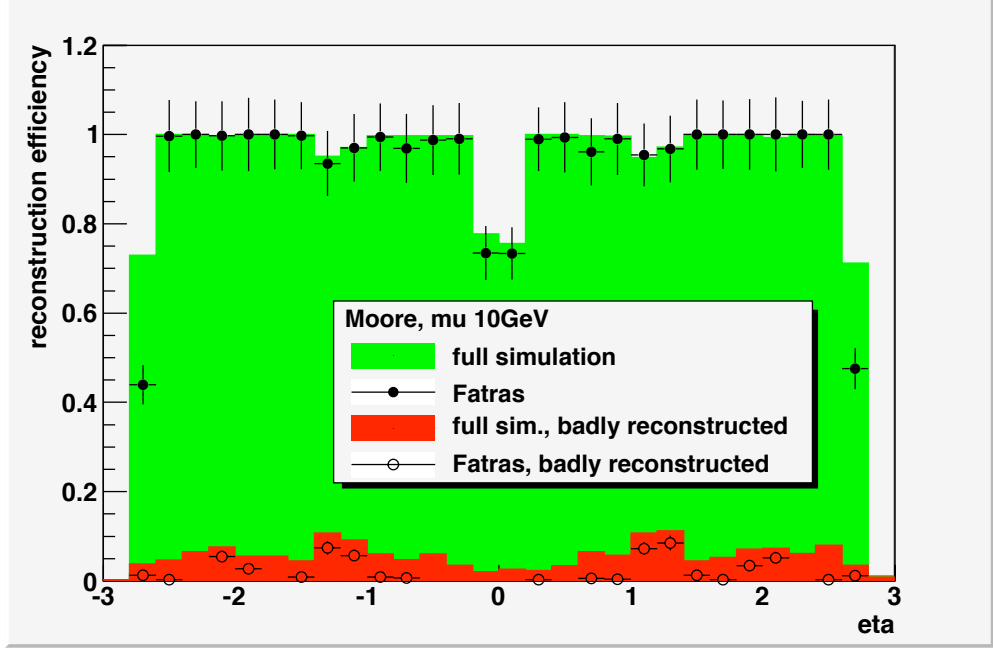




\section{atras}

Šárka Todorova

IEEE2010

Introduction

Motivation

Fatras ID

Fatras MS

Fatras Combined

Validation \&

performance

Summary

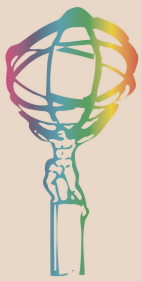

11

\section{ATLAS Calorimeter}

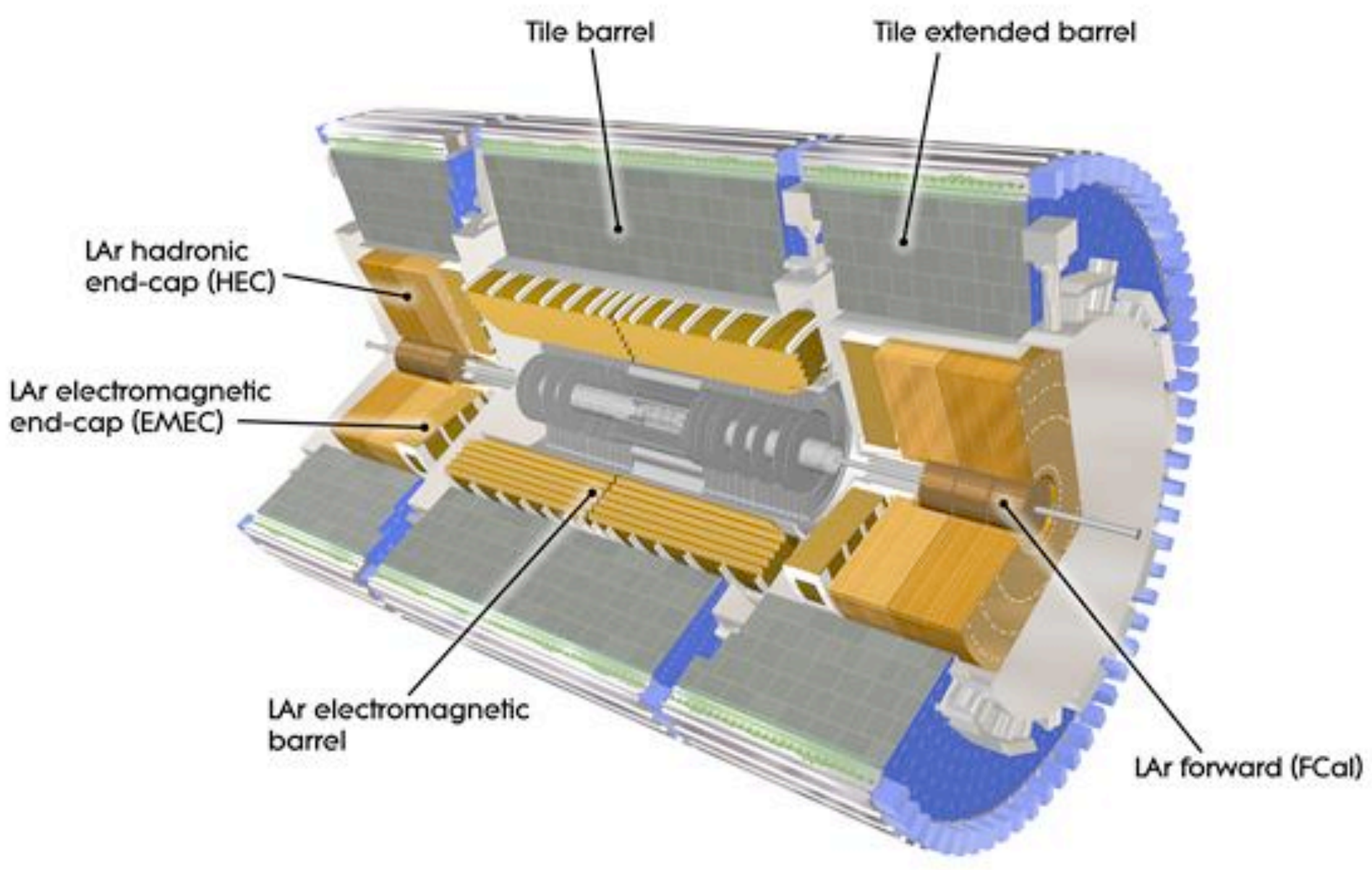




\section{Combined FATRAS : simulating Calo}

\section{atias}

Muon transport through Calo ( $<1 \mu \mathrm{s})$

- energy deposit collected

- mean energy loss underestimates 'catastrophic energy loss'

hard bremstrahlung

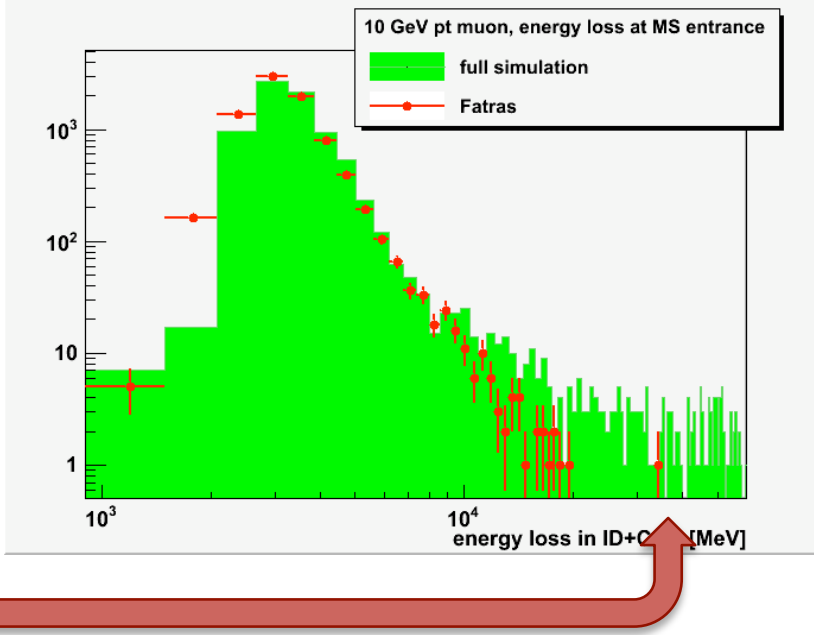

IEEE2010

Introduction

Motivation

Fatras ID

Fatras MS

Fatras Combined

Validation \&

performance

Summary
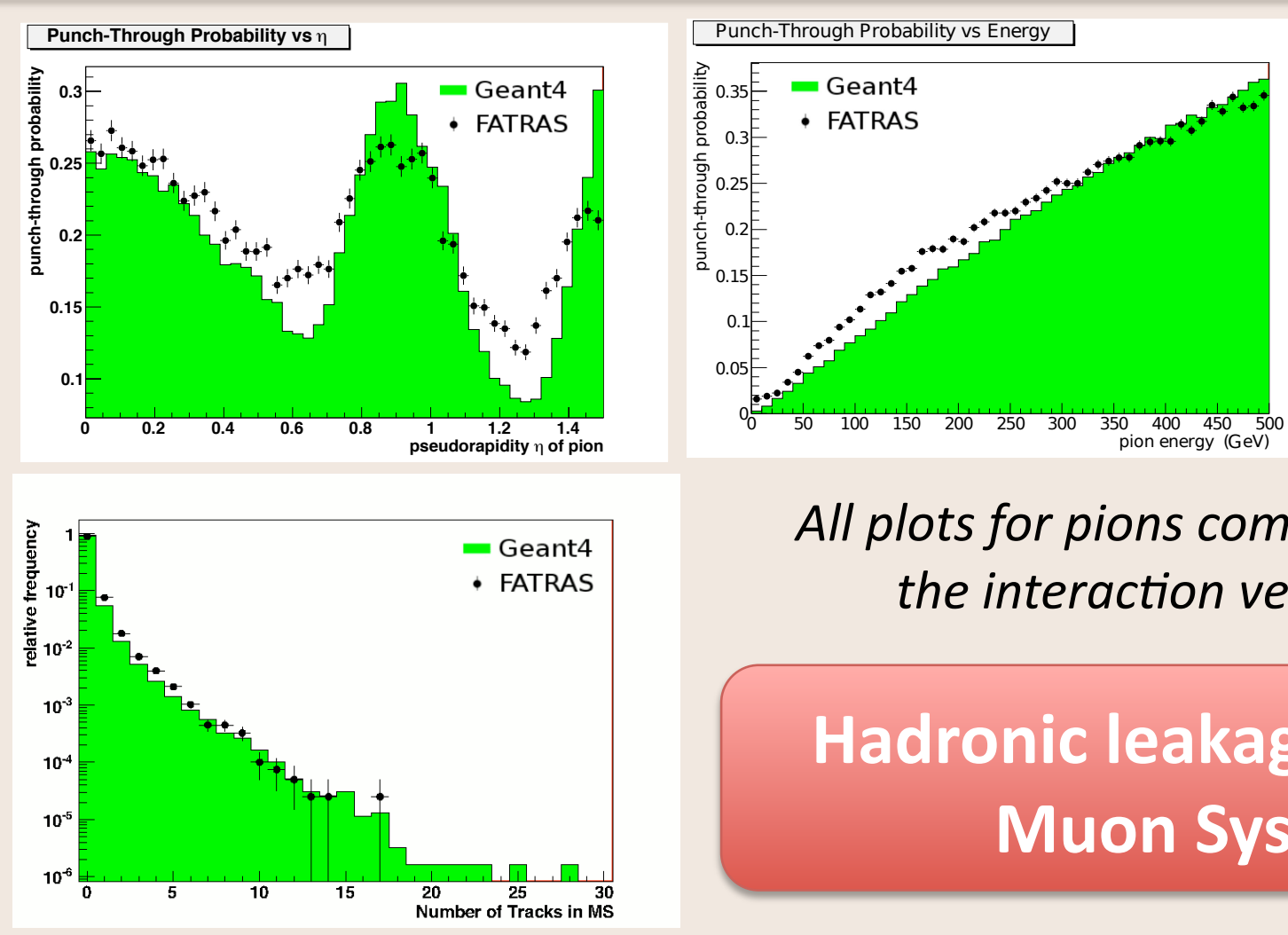

All plots for pions coming from the interaction vertex

Hadronic leakage into the Muon System 


\section{Combined FATRAS : simulating Calo}

Interface to FastCaloSim:

Šárka Todorova

IEEE2010

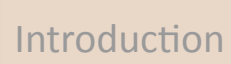

Motivation

Fatras ID

Fatras MS

Fatras Combined

Validation \&

performance

Summary
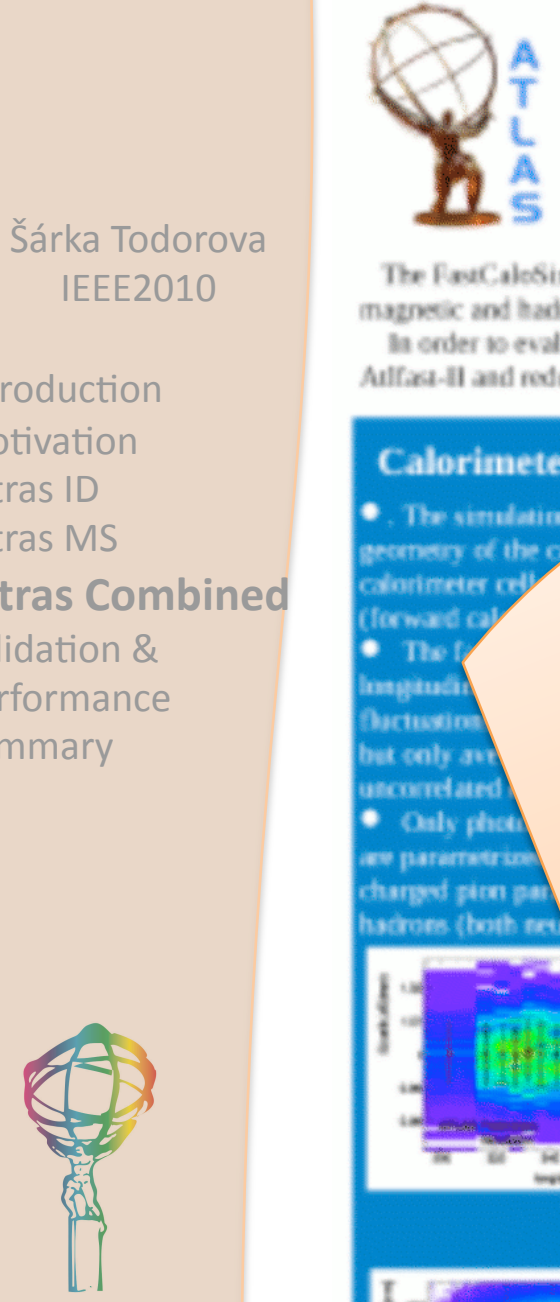

2010 Nuclear Science Symposium, in Knoxville, U.S.A., 1-5 November 2010

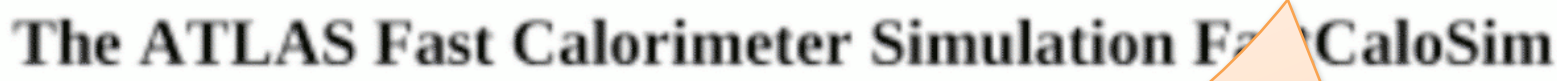

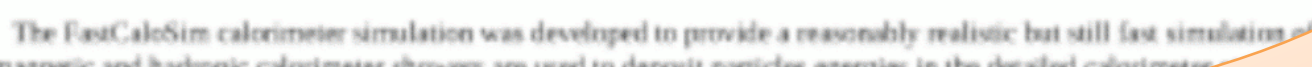

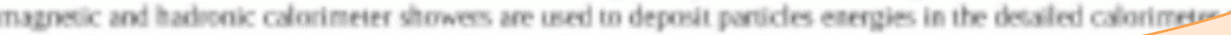

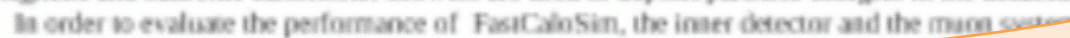

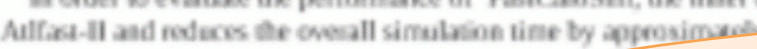

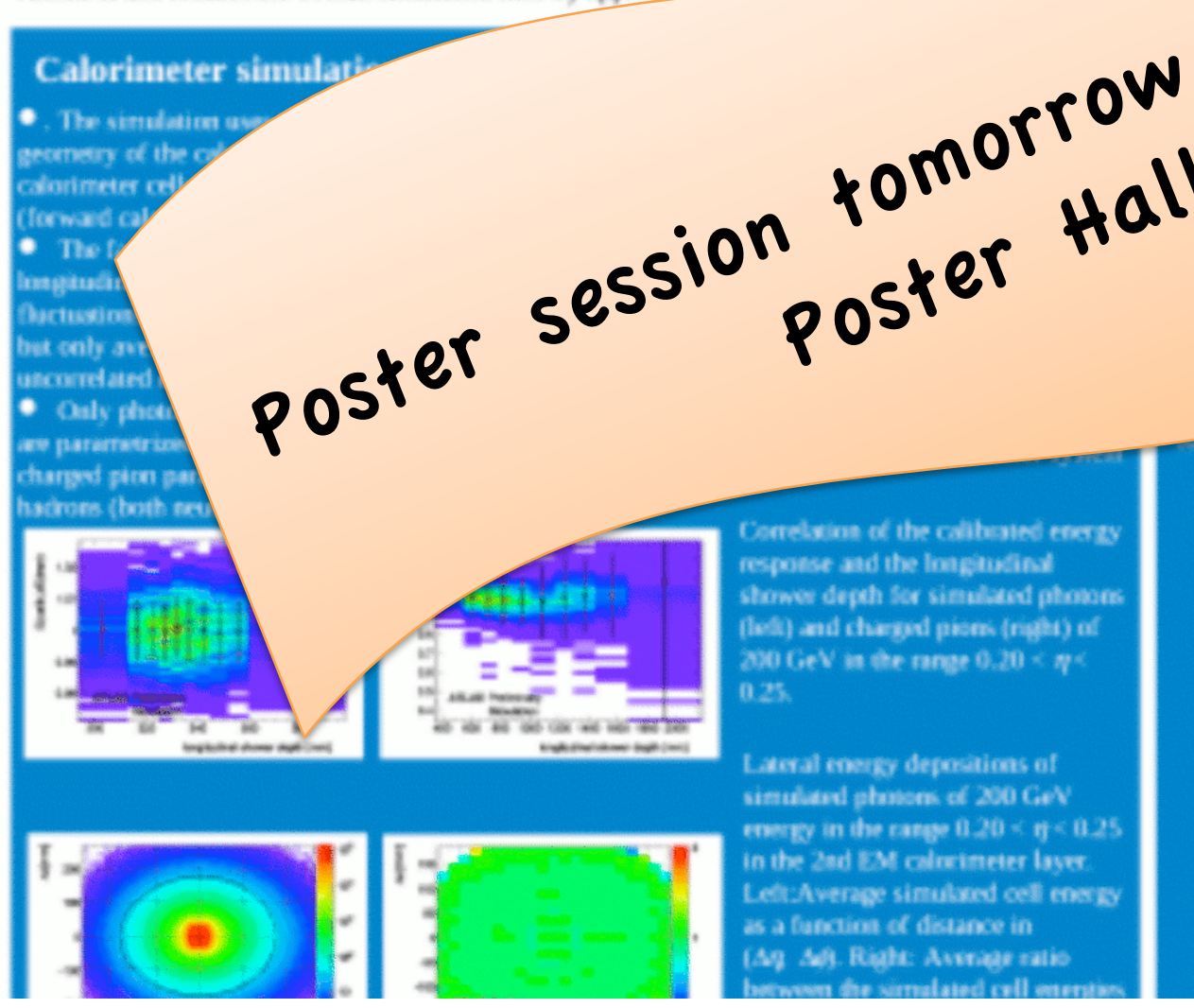
morning

pem. Paramericasines of electimIll and fast simulaine is called 


\section{fatias \\ FATRAS validation: minbias sample (5k events)}
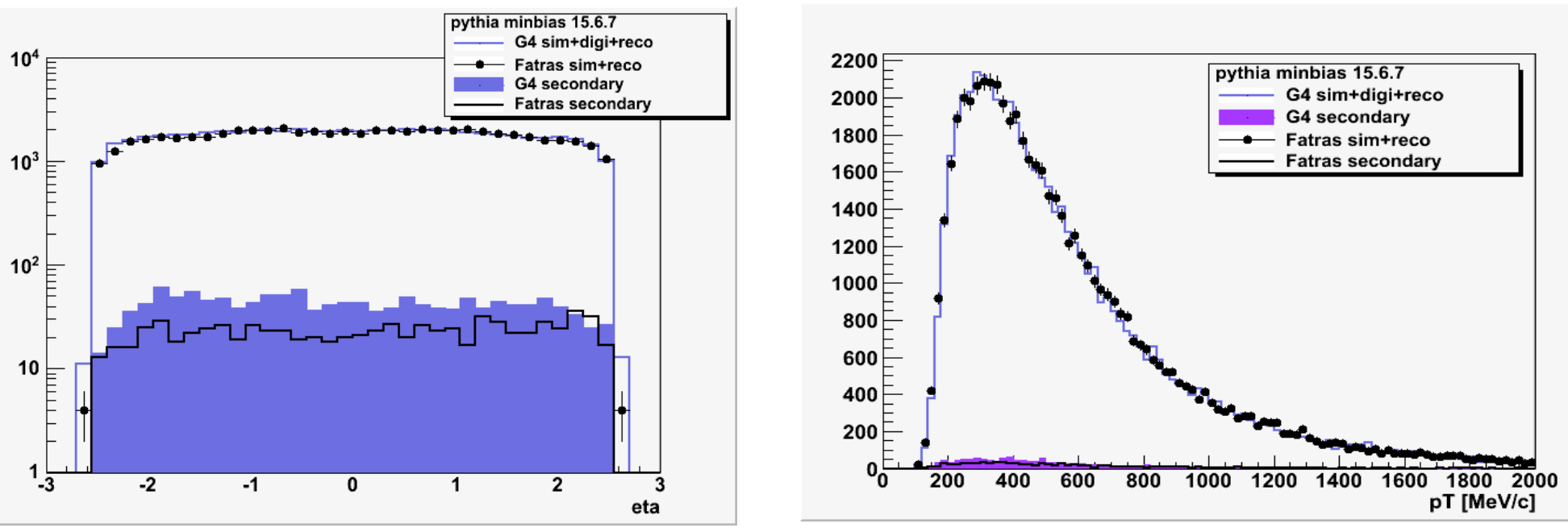

Fatras ID

Fatras MS

Fatras Combined

Validation \&

performance

About 1\% of secondaries missing in Fatras

- mainly leptons - traced to photon

Summary

\section{conversion \& pion decay}

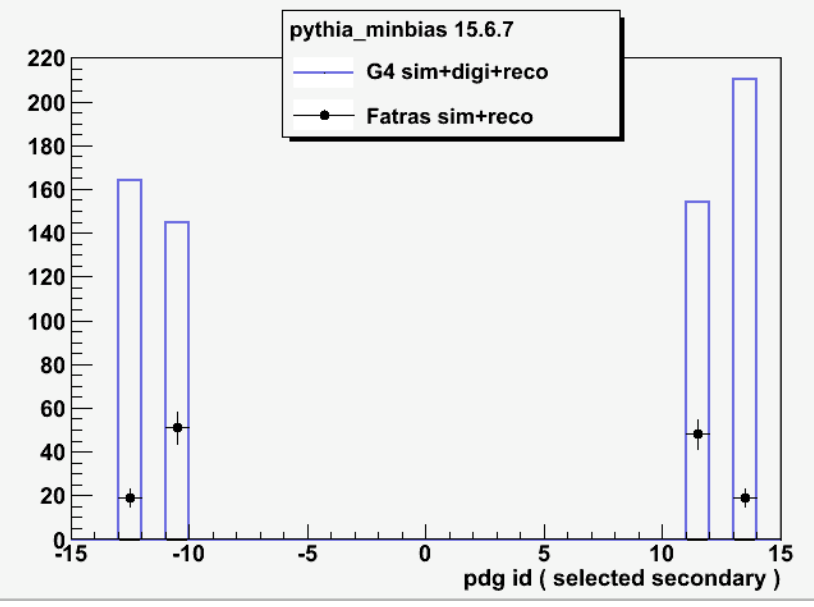

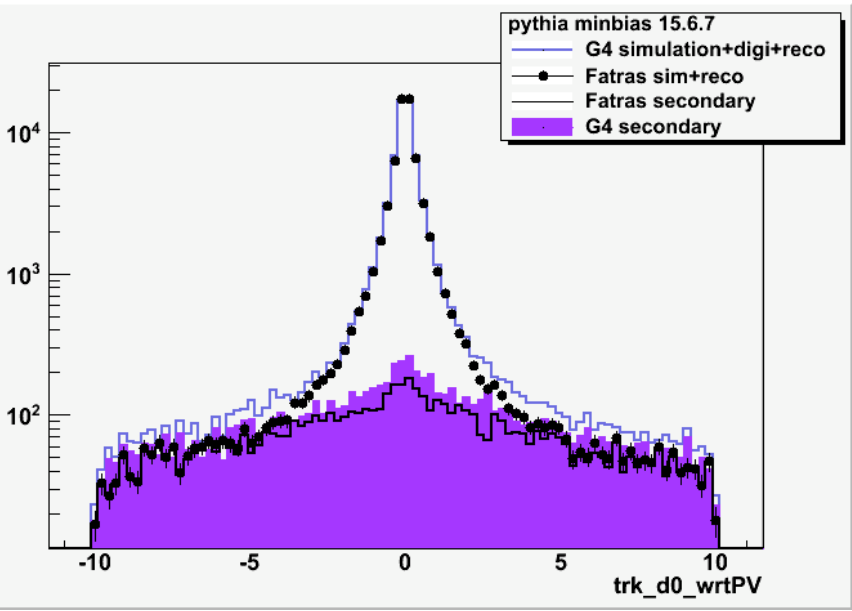

impact parameter distribution narrower due to primary track reconstruction 


\section{fatias}

\section{FATRAS validation: combined muons}

Reconstructed ZO mass ( Z-> $\mu \mu$ sample)
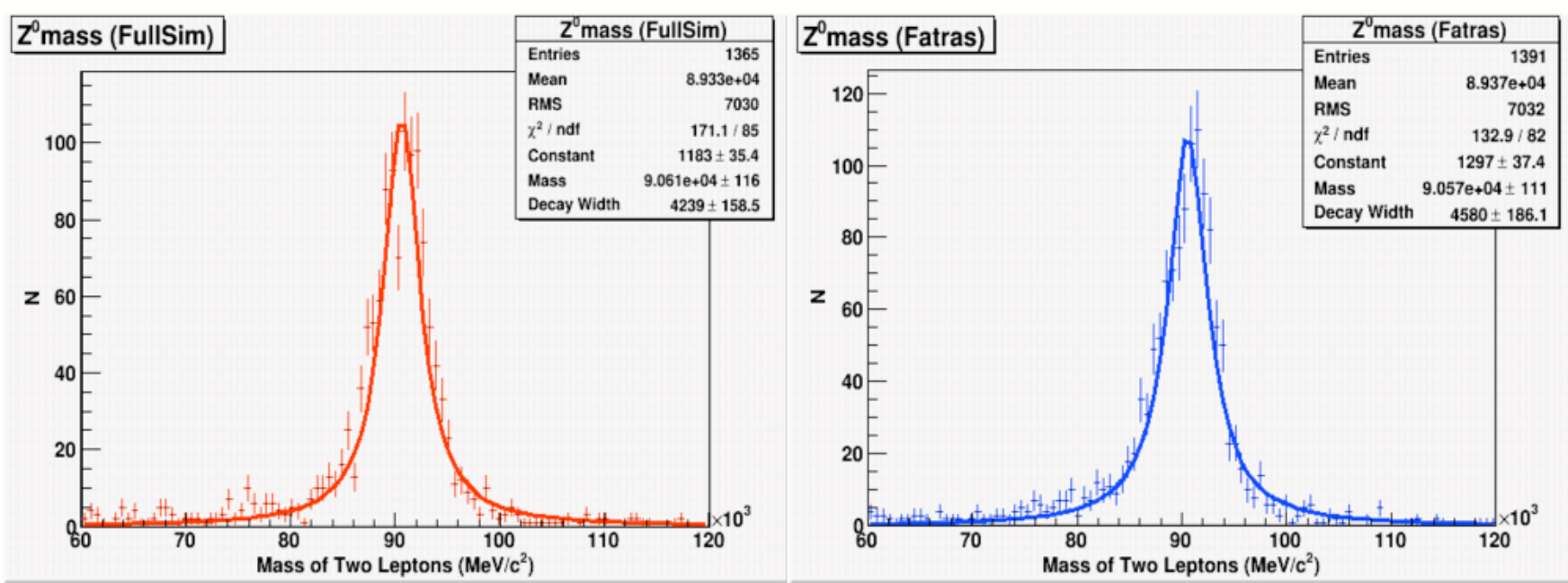

Reconstructed di-muon mass ( tt sample ), using full reconstructed error matrix

performance

Summary

IEEE2010

Introduction

Motivation

Fatras ID

Fatras MS

Fatras Combined

Validation \&

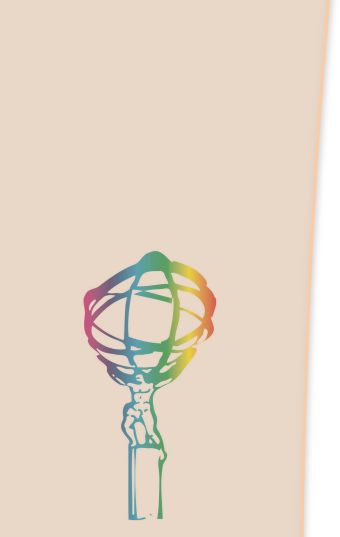

15
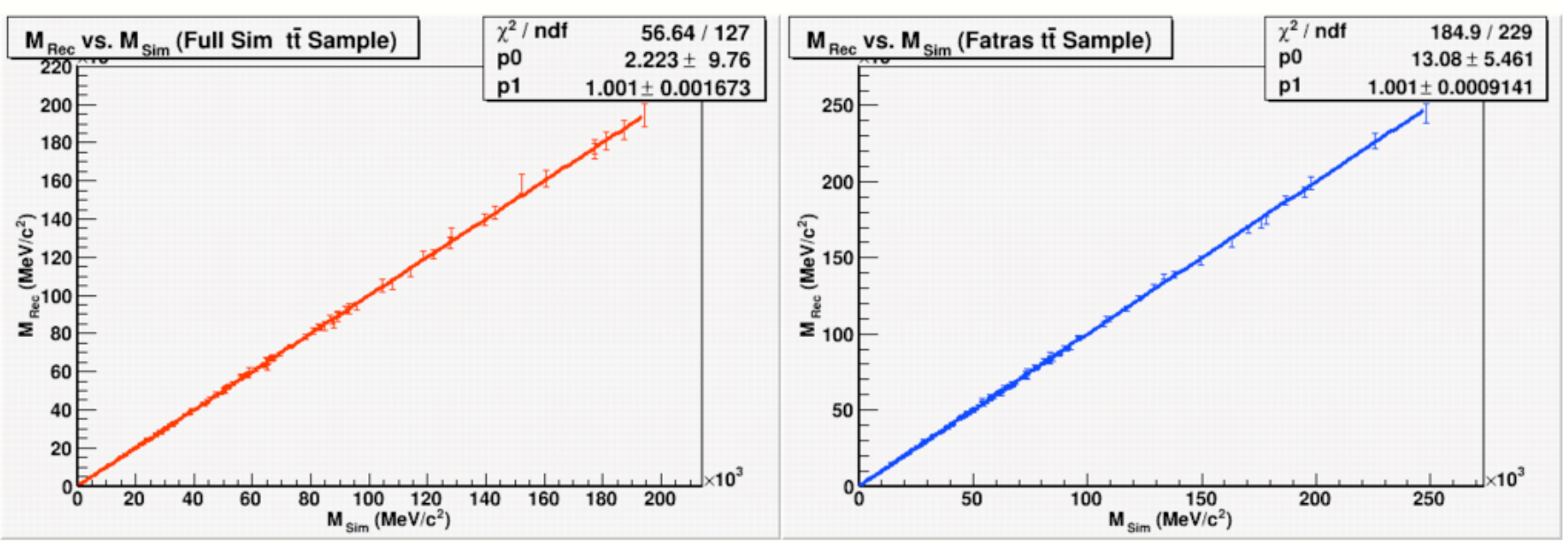


\section{FATRAS project: performance}

How fast we really are?

Šárka Todorova

IEEE2010

Introduction

Motivation

Fatras ID

Fatras MS

Fatras Combined

Validation \&

performance

Summary

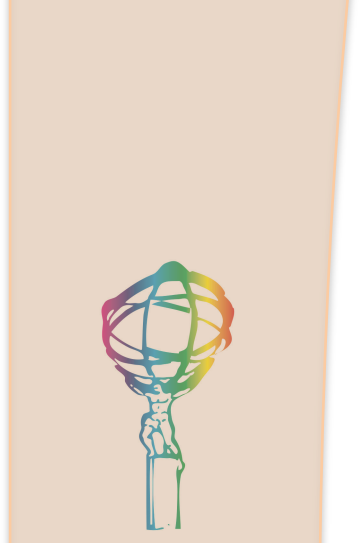

16

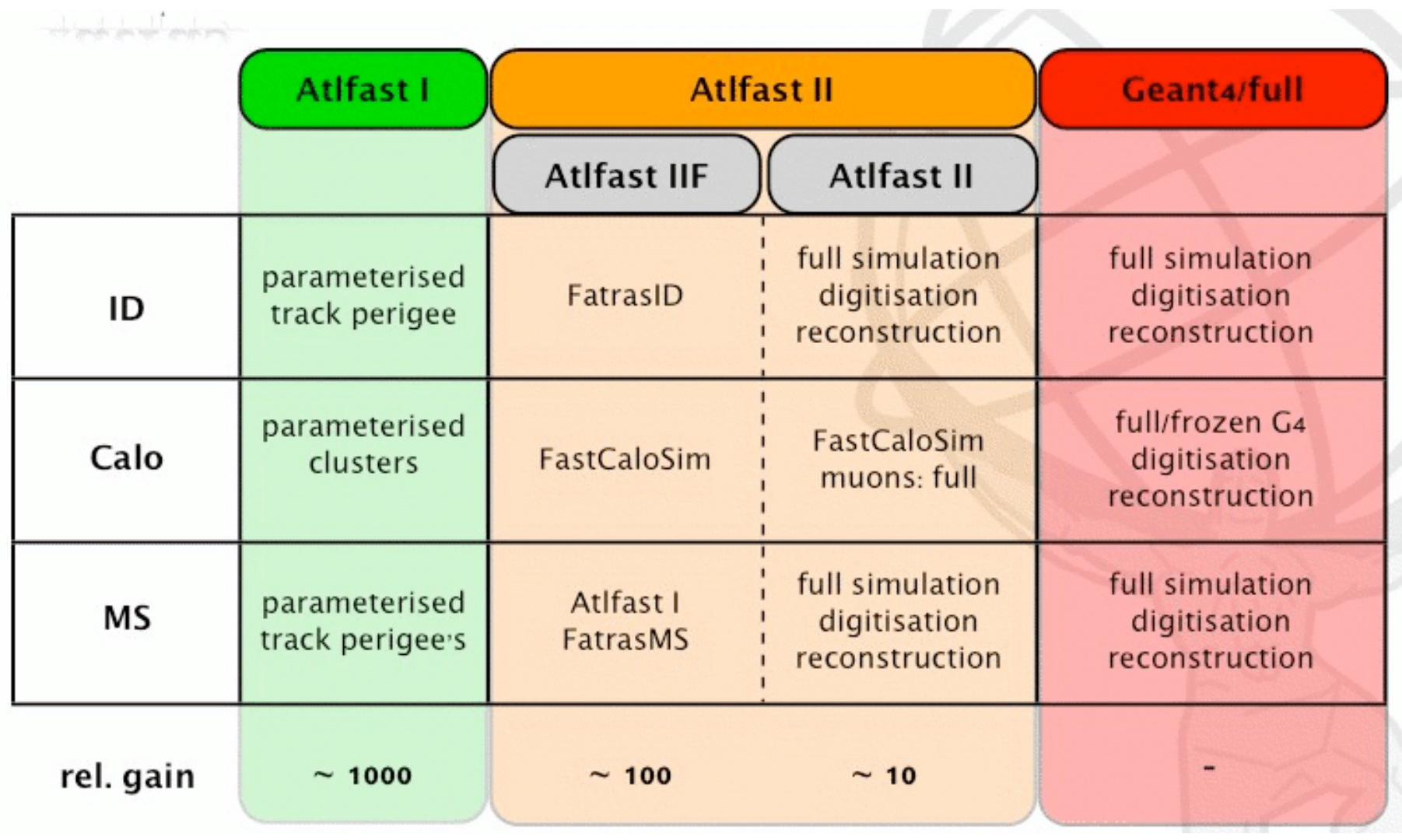


fatias

Šárka Todorova

IEEE2010

Introduction

Motivation

Fatras ID

Fatras MS

Fatras Combined

Validation \&

Summary

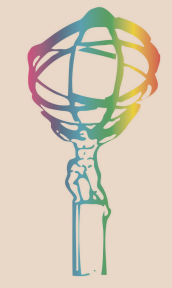

17

\section{Summary}

fatlas is a tool for the future

Succesfully validated on number of physics analyses,

\& with first ATLAS real data

Numerous applications :

- fast evaluation of impact of modified detector status

on a given analysis

- study of detector design ( LHC upgrade)

- study of misaligned geometry

$-\ldots$

Number of ongoing (sub)projects:

- overlay of Fatras (background) samples with signal MC

- interface to Geant4 libraries ( detailed parametrization of hadronic interactions) 